\title{
虚拟恒电位仪的研制及其在腐蚀电化学测量中的应用
}

\author{
孙风娟王 佳 ${ }^{*}$ \\ (中国海洋大学化学化工学院, 山东青岛 266100; 金属腐蚀与防护国家重点实验室, 沈阳 110015)
}

\begin{abstract}
摘要: 恒电位仪是腐蚀电化学测量最重要的基本设备. 虚拟恒电位仪用图形程序软件取代复杂硬件来实现恒 电位功能. 它的结构和参数灵活, 构建简单, 能满足多方面腐蚀电化学测试方法的需求, 虚拟恒电位仪的实现是 发展虚拟腐蚀电化学仪器的重要步骤. 本文采用 LabVIEW 2010 编程系统和比例-积分-微分(PID)控制技术, 研 制了虚拟恒电位仪, 并组建了 VEC11-A 腐蚀电化学测试系统, 该系统能满足不同阻抗体系的腐蚀电化学测试 需求. 其腐蚀电位、稳态极化曲线、线性极化曲线、循环伏安、动电位扫描、恒电位阶跃等常规腐蚀电化学测量结 果与 2263 电化学测试系统相近, 能够满足常规腐蚀电化学测试要求, 具有很大的发展空间.
\end{abstract}

关键词: 虚拟仪器; 稳态极化曲线; 恒电位仪; PID 控制; LabVIEW

中图分类号： O646; TG174.3

\section{Development and Application of Virtual Potentiostat on Electrochemical Corrosion Measurement}

\author{
SUN Feng-Juan WANG Jia* \\ (College of Chemistry and Chemical Engineering, Ocean University of China, Qingdao 266100, Shandong Province, P. R. China; \\ State Key Laboratory for Corrosion and Protection of Metals, Shenyang 110015, P. R. China)
}

\begin{abstract}
A potentiostat is a fundamental piece of equipment for the study of corrosion electrochemistry and research into the application of virtual technology to potentiostats is becoming increasingly important. The functions of virtual potentiostats can be controlled and implemented using software instead of complex hardware. They would be capable of not only meeting the demands of a variety of electrochemical tests, but would also be more flexible offering a simpler structure and operating parameters. This would represent a significant development in virtual electrochemical corrosion instrumentation. In this paper, a virtual potentiostat measurement system VEC11-A (virtual electrochemical corrosion test system) has been designed based on LabVIEW. The virtual instrument system of the potentiostat, based on the proportionintegration-differentiation (PID) control technology operated under the LabVIEW 2010 programming platform, was used to determine corrosion potential measurement, polarization curves, linear polarization curves, cyclic voltammetry curves, dynamic potential anode passivation curves, and constant potential step curves. In contrast with normal electrochemical instruments, the system worked well for electrolyzers of different impedances.
\end{abstract}

Key Words: Virtual instrument; Steady-state polarization curve; Potentiostat; PID control; LabVIEW

\section{1 引言}

软件化、网络化和总线标准化是未来测试仪器
技术发展的主要方向. 虚拟仪器“软件就是仪器”的 思路是在测试系统和仪器设计使用软件实现硬件

Received: October 19, 2011; Revised: December 30, 2011; Published on Web: January 10, 2012.

"Corresponding author. Email: jwang@ouc.edu.cn; Tel: +86-532-66781903.

The project was supported by the National Natural Science Foundation of China (50971118, 51131005).

国家自然科学基金(50971118, 51131005)资助项目

(C) Editorial office of Acta Physico-Chimica Sinica 
的功能, 既降低了成本, 又提高了仪器的灵活性和 扩展能力. ${ }^{1}$ LabVIEW 图形编程系统是发展虚拟仪 器的重要方法. 它提供了大量的功能模块, 为不具 备程序语言基础的科研工作者提供了构建拥有复 杂计算解析功能的测试系统的简单而高效的程序 方法. ${ }^{2}$ 随着近年来各个领域中虚拟仪器技术的快速 发展, 测试仪器已经开始进入虚拟仪器时代.

相比之下, 腐蚀电化学领域中的测试仪器仍以 硬件为主, 虚拟仪器技术发展较慢. 其主要问题是 虚拟恒电位仪技术未得到发展. 近年来, 部分虚拟 电化学仪器技术已取得了一些成果. ${ }^{3}$ 潘国运等 ${ }^{4}$ 研 制了虚拟频率响应分析仪+恒电位仪, 并进行了腐 蚀电化学阻抗测量, 在不同阻抗体系中均得到了准 确的结果. $\mathrm{Li}$ 等 ${ }^{5}$ 利用虚拟仪器技术构建多通道电化 学检测系统, 使用八个自制的由 $9 \mathrm{~V}$ 电池带动的恒 电位仪, 可以四个通道同时工作, 完成循环伏安、电 流分析等功能. Perusse 和 Leech ${ }^{6}$ 在利用 LabVIEW 编程来控制实现线性扫描和循环伏安测试系统中 使用了硬件双恒电位仪; Economou等 ${ }^{7}$ 采用部分虚 拟仪器技术进行阳极和阴极方波电位扫描、吸附伏 安测试以及富集方波伏安测试也是基于两个自制 的硬件恒电位仪; 韩否等 ${ }^{8,9}$ 所研制的基于虚拟仪器 技术的便携式电化学检测系统中采用的是商品化 的恒电位仪和自制的硬件反相放大式恒电位. 目前 为止, 电化学领域中的虚拟仪器测试系统仍离不开 硬件恒电位仪, ${ }^{10}$ 只能称之为部分虚拟电化学仪器. 因此, 发展完全虚拟电化学测试仪器的关键在于虚 拟恒电位仪技术.

为此, 本文采用 LabVIEW 虚拟仪器技术研制了 以 VEC11-A (Virtual Electrochemical Corrosion Test System)虚拟恒电位仪为主体的腐蚀电化学测试系 统, 并与美国 AMETEK 公司的 2263 恒电位仪进行 对比来实现该体系的测试和评价.

\section{2 虚拟恒电位仪的设计}

\section{1 恒电位仪的恒定极化电位原理}

电化学测量是一种扰动方法, 即向待测体系输 入电化学扰动信号后测量体系对扰动的电化学响 应. 为了获得解析的响应结果, 扰动信号应严格按 指定函数方式变化, 最常用的扰动是电位扰动, 实 现这一功能的仪器是恒电位仪. 因此, 恒电位仪成 为电化学测量最基本仪器. 恒电位仪的工作原理是 极化电位信号通过辅助电极 $\mathrm{C}$ 施加在工作电极上,
同时, 由参比电极 $\mathrm{R}$ 测量工作电极 $\mathrm{W}$ 的电位 $V_{\mathrm{w}}$, 测 量结果输入恒电位仪后, 仪器立即比较测定的 $V_{\mathrm{w}}$ 和 设定电位 $V_{\mathrm{f}}$ 之间的偏差, 并根据这一偏差自动调节 极化电流来减小该偏差. 例如, 若 $V_{\mathrm{w}}>V_{\mathrm{f}}$, 则减小极 化回路电流, 使两者偏差减小. 反之, 若 $V_{\mathrm{w}}<V_{\mathrm{f}}$, 则增 加电流来使偏差减小, 直至 $V_{\mathrm{w}}$ 和 $V_{\mathrm{f}}$ 的偏差小于预设 偏差值 (如 $0.2 \mathrm{mV}$ ), 恒电位过程完成. 并在整个测试 中持续这一调节过程, 保持 $V_{\mathrm{w}} \approx V_{\mathrm{f}}$. 其调节过程如图 1 所示.

图 1 中恒电位仪向 C-W 提供一初始电流, $\mathrm{R}$ 测 定 $\mathrm{W}$ 的电位后输入恒电位仪反相端, 恒电位仪比较 两端电位差后调节输出到 $\mathrm{C}$ 的电位, 使 $\mathrm{W}$ 电位接近 设定电位 $V_{\mathrm{f}}$, 恒电位仪比较两端电位差后再次调节 输出到 $\mathrm{C}$ 的电位, 直至 $\mathrm{W}$ 电位与设定电位 $V_{\mathrm{f}}$ 差值小 于预设偏差, 达到恒定 $\mathrm{W}$ 电位的目的. 这是一个闭 环负反馈系统, 其恒定电位的速度和精度取决于恒 电位仪内的比较调节性能. 一般的恒电位仪都是采 用运算放大器来实现这一功能, 通常采用电压跟随 器和反向放大器形式. 在电压跟随器电路中, 基准 电位讯号加在运算放大器的同相输入端, 而参比电 极电位作为反馈讯号加入反向输入端, 根据运算放 大器同相输入时的跟随特性, 参比电极的电位随着 基准电位而变化且能自动调节以达恒定; 在反向放 大器形式电路中, 基准电位和参比电位分别通过输 入电阻与反馈电阻接入反相输入端, 输入电阻与反 馈电阻相等, 同样能达到自动调节电位的目的. ${ }^{11}$

\section{2 虚拟恒电位仪的设计思路}

VEC11-A 虚拟恒电位仪由计算机、具有模拟 输入与模拟输出功能的板卡和软系统件组成. 板卡 完成 $\mathrm{W}$ 电位采集和 $\mathrm{C}$ 电位加载的功能. 软件完成 上述硬件恒电位仪所需完成的一系列比较和调节 输出电位的功能. 虚拟恒电位仪省略了昂贵的仪器

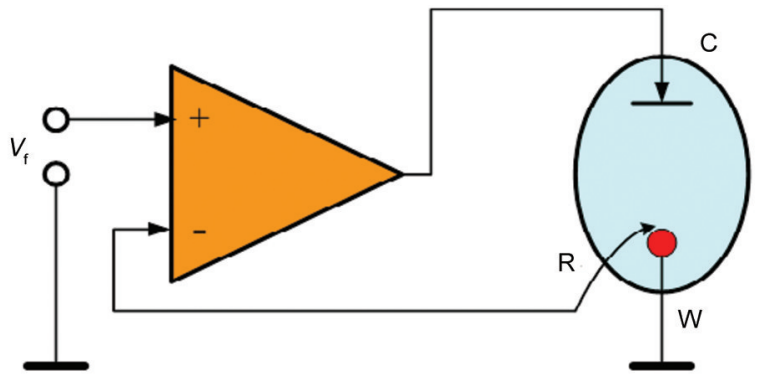

图 1 恒电位仪工作原理示意图

Fig.1 Schematic diagram of the operating principle of potentiostat

$V_{\text {f: }}$ setting potential; W: working electrode; C: counter electrode; R: reference electrode 
硬件, 代之以软件实现恒电位功能, 其优越性显而 易见. 其性能取决于软件的采样-比较-调节-采样比较…这一闭环的性能, 调节次数越少, 恒电位速 度越快; 预设偏差越小, 恒电位精度越高, 而调节的 次数就越多. 根据采样值输出合适的极化电位是关 键. 理想的情况是一次调节到位, 但由于体系板卡 性能和计算机性能存在延迟, 调节方式和参数通常 需要根据体系硬件系统响应特性来改变和优化至 最佳状态. 通常的比例调节很难达到较高的性能.

比例-积分-差分(PID)调节方法是自动控制领域 中最常用的高性能反馈调节技术. 其调节原理如下.

电源向被测系统施加一定的电压信号, 同时测 量要求恒压部分的电压, 通过初始信号与响应信号 的关系, 设置一定参数进行比例、微分和积分调节, 调节后输出电压作为下一次初始电压. 整个程序的 核心就是 PID 控制, 其原理如图 2 所示.

其中的关键步骤是 PID 控制, PID 控制是一个 在工业控制中广泛使用的反馈控制回路. 这个回路 把收集到的数据和一个参考值进行比较, 然后把这 个差别用于计算新的输入值, 这个新的输入值的目 的是可以让系统的数据达到或者保持在参考值. 和 其他简单的控制运算不同, PID 控制器可以根据历 史数据和差别的出现率来调整输入值, 这样可以使 系统更加准确, 更加稳定. 图 2 中, $r(t)$ 为要求的参考
值; $u(t)$ 为经过 PID 计算产生的新输入值, 也就是加 在被测系统中的值; $c(t)$ 为恒定部分的实际测量值; $e(t)$ 为收集到的数据 $c(t)$ 与参考值 $r(t)$ 的比较值; PID 算法就是根据 $e(t)$ 的值计算 $u(t)$, 如下式:

$$
u(t)=k_{\mathrm{p}}\left[e(t)+\frac{1}{T} \int_{0}^{\tau} e(\tau) \mathrm{d} \tau+T_{\mathrm{d}} \frac{\mathrm{d} e(t)}{\mathrm{d} t}\right]
$$

式中, $k_{\mathrm{p}}$ 为比例系数; $T$ 为积分时间常数; $T_{\mathrm{d}}$ 为微分时 间常数, 这三个参数的整定是控制系统设计的核心 部分, 也是最复杂的部分. ${ }^{12-15}$ 图 3 为本系统的程序 流程图.

经过多次测试和分析, 获得了适合于本机 VEC11-A 虚拟恒电位仪系统 PID 参数.

\subsection{VEC11-A 虚拟恒电位仪系统的设计}

VEC11-A 虚拟恒电位仪系统结构如图 4 所示, 由计算机、多功能采集卡及控制软件组成. 其中, $R$ 为 采样电阻, 作用是差分输入采集电流. 数据采集卡为 $\mathrm{NI}$ 公司(美国)M系列 PCI-6251多功能采集卡. 软件 编程采用 NI 开发的图形编程平台 LabVIEW2010. 如前所述, 虚拟恒电位仪的性能取决于控制软件, 在开发平台上, 按照模块化思想设计系统软件. 主 要有初始数组模块、读写存储设置模块、数据采集 模块, ${ }^{16-20}$ 时间设置模块以及 PID 调控模块. 在初始 数组模块生成阶梯数组; 在读写存储设置模块对一 个模拟输出通道和两个模拟输入通道进行初始设

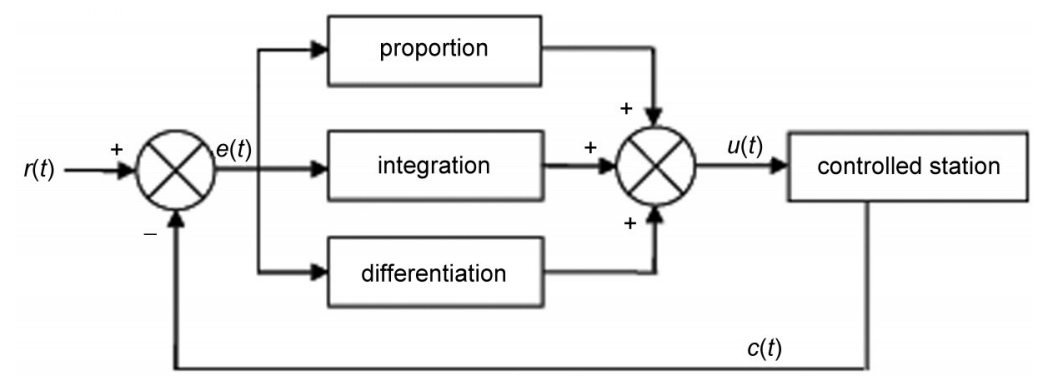

图 2 PID 控制原理图

Fig.2 Diagram for PID control

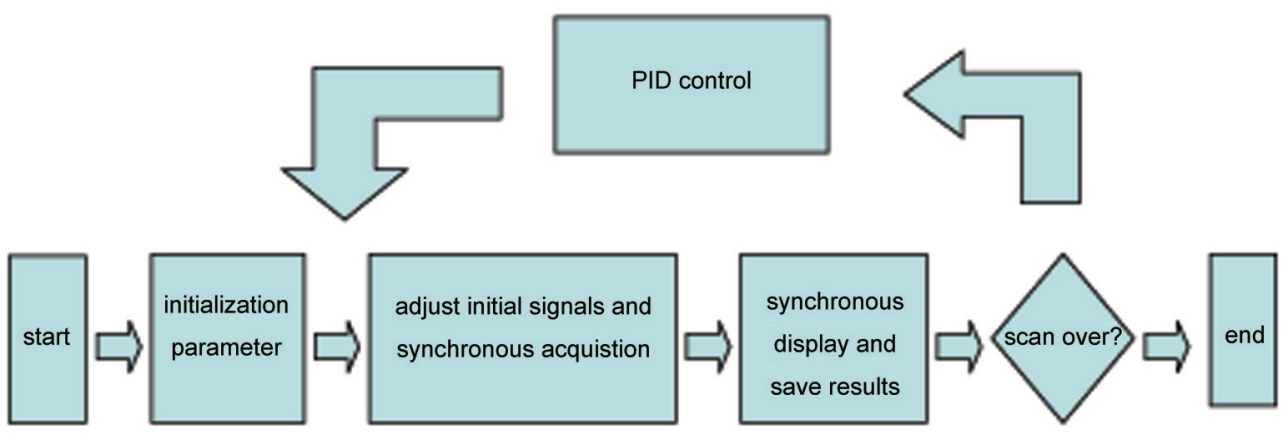

图 3 测试系统流程图

Fig.3 Flowchart for the testing system 


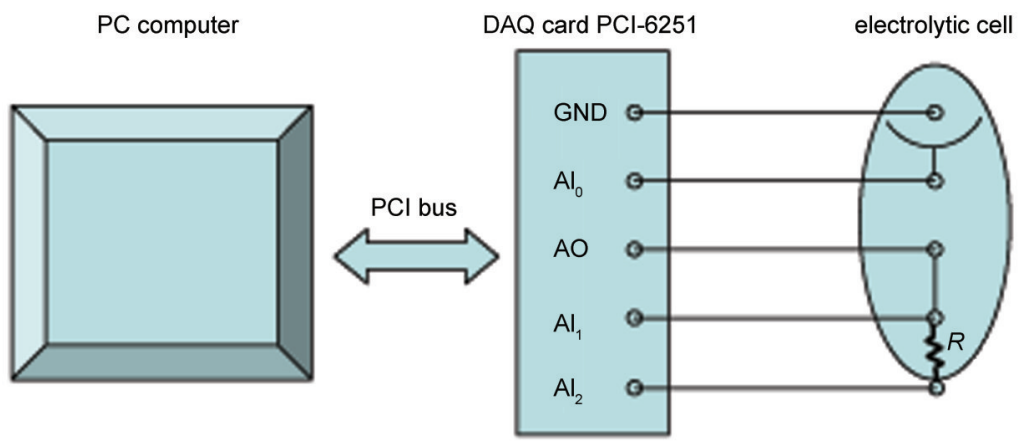

图 4 测试系统构成图

Fig.4 Constitution of the testing system

置; 时间设置模块对程序运行时间及阶跃稳定时间 进行控制; PID 调节模块中, 根据采集到的数据及 PID 运算法则算出合适的输出电压; 采集加在采样 电阻上的电压换算为电流, 最后以电位对时间关系 图和电位对电流关系图显示测量结果, 并以文本格 式保存.

\section{3 结果与讨论}

\subsection{VEC11-A 虚拟恒电位仪的测量功能}

研制的 VEC11-A 虚拟腐蚀电化学测试系统具 有通用腐蚀电化学测试系统的常规测试功能. 包括 测量腐蚀电位、稳态极化曲线、线性极化曲线、循环 伏安、动电位扫描、恒电位阶跃等功能, 能够满足简 单的腐蚀电化学测试要求. ${ }^{21,22}$
为检验研制虚拟仪器的性能, 分别对模拟电解 池和真实电化学体系进行测量, 并与美国 AMETEK 公司的 PARSTAT 2263 恒电位仪 c(以下简称 2263) 测试结果进行对比.

\section{2 电子元件模拟电路 VEC11-A 虚拟恒电位仪实} 测结果

模拟电解池的等效电路如图 5 所示. 其中 $R=$ $0-2 \mathrm{M} \Omega, R_{1}=990 \Omega, R_{2}=990 \Omega, R_{3}=10 \Omega, C=100 \mu \mathrm{F}$.

为了验证系统的响应速度及精确度, 对模拟电 路施加-700 mV 恒电位极化. 测量结果见图 6(A), VEC11-A 虚拟恒电位仪测定的电位为 $(-700 \pm 0.05)$ $\mathrm{mV}$, 与极化电位值完全一致, 电位波动小于 0.05 $\mathrm{mV}$, 具有较高的电位跟随精度.

然后, 在模拟电解池中在 -100 到 $100 \mathrm{mV}$ 范围

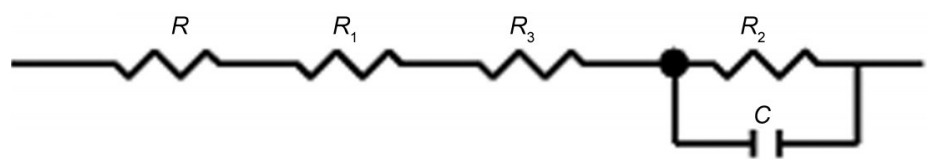

图 5 模拟全电解池等效电路

Fig.5 Equivalent electrical circuits of the simulated electrolytic cell

$R$ : variable resistance; $R_{1}$ : sampling resistance; $R_{2}$ : working resistance; $R_{3}$ : resistance of solution; $C$ : capacitance of the electric double layers
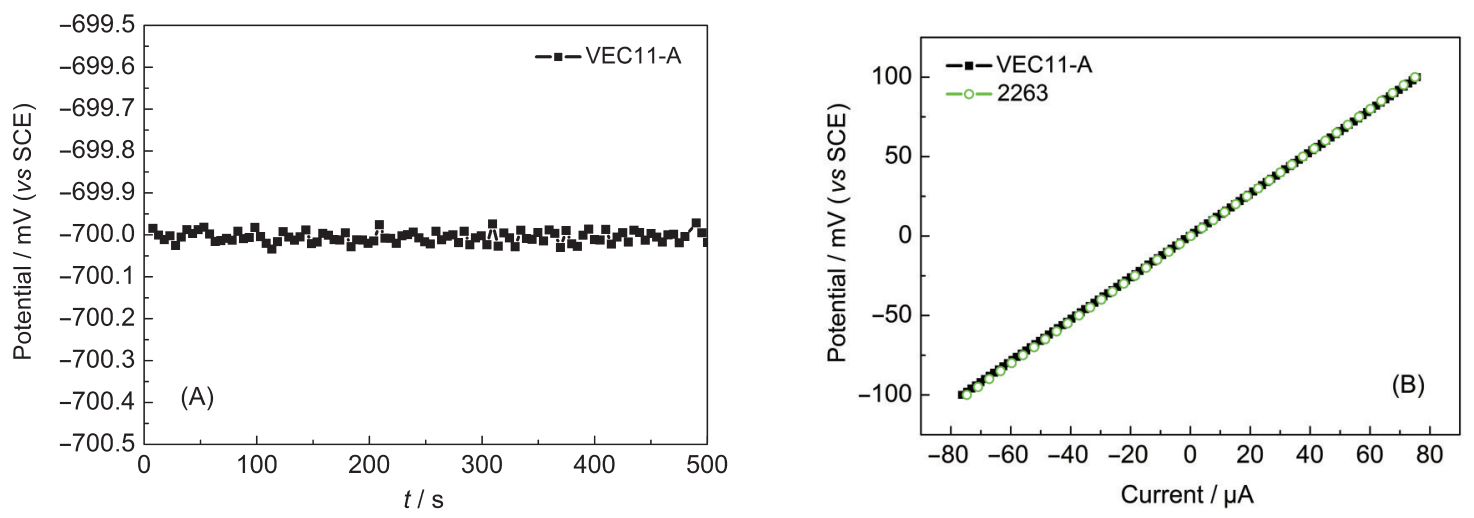

图 6 VEC11-A 虚拟恒电位仪和 2263 恒电位仪在 $-700 \mathrm{mV}$ 恒电位(A)和在 $-100-100 \mathrm{mV}$ 之间扫描速率为 $0.5 \mathrm{mV} \cdot \mathrm{s}^{-1}$ 时的 动电位(B)测量结果比较

Fig.6 Result comparisons of VEC11-A vitual potentiostat and 2263 potentiostat at the potential of $-700 \mathrm{mV}$ (A) and dynamic potential scan from -100 to $100 \mathrm{mV}$ with a scan rate of $0.5 \mathrm{mV} \cdot \mathrm{s}^{-1}$ (B) 
施加间隔 $5 \mathrm{mV}$ 扫描阶梯电位, 每个阶梯稳定 $10 \mathrm{~s}$. 测定结果如图 6(B)所示, VEC11-A 虚拟恒电位仪和 2263 恒电位仪测定的结果完全一致.

\section{3 实际电化学体系的 VEC11-A 虚拟恒电位仪测 量结果}

Q235 碳钢工作电极电极表面用金相砂纸逐级 打磨至 1000 \#, 然后丙酮除油和蒸馏水清洗. 实验 采用三电极体系, 参比电极为饱和甘录电极( $\mathrm{SCE}$ ), 辅助电极为不锈钢网电极. 电极工作面积均为 1 $\mathrm{cm}^{2}$.

\subsection{1 腐蚀电位测量}

腐蚀电位测量部分是恒电位仪的最基本的功 能, 为了验证这一功能, 对 $\mathrm{Q} 235$ 碳钢在质量分数 (下同)为 $3.5 \%$ 的 $\mathrm{NaCl}$ 溶液中的三电极体系进行测 量, 从图 7 可以看出, 在长达 $6000 \mathrm{~s}$ 的时间内, VEC11-A 系统与 2263 恒电位仪测量结果一致, 基本 符合测试要求.

\subsection{2 恒电位测量}

为了验证系统的响应速度及精确度, 对 Q235 碳钢在海水中的三电极体系进行测量: 对三电极体 系施加 $-100 \mathrm{mV}$ 电位并测量结果 $(-100 \mathrm{mV}$ 恒定电 位). 如图 8 所示, VEC11-A 虚拟恒电位仪测定的电 位波动范围为 $\pm 0.5 \mathrm{mV}$, 与图 6(A)类似, 其平均值与 标准数据一致。

\subsection{3 稳态极化曲线测量}

稳态极化曲线是实验室最常使用的腐蚀行为 测量技术. ${ }^{23}$ 为此采用 VEC11-A 虚拟恒电位仪和 2263 恒电位仪进行了对比测量, 以检验研制的虚拟 仪器的测量性能.

图 9(A), (B), (C), (D) 分别为碳钢电极在 0.5 $\mathrm{mol} \cdot \mathrm{L}^{-1}$ 的 $\mathrm{H}_{2} \mathrm{SO}_{4}$ 溶液、碳钢电极在 $3.5 \%$ 的 $\mathrm{NaCl}$ 溶

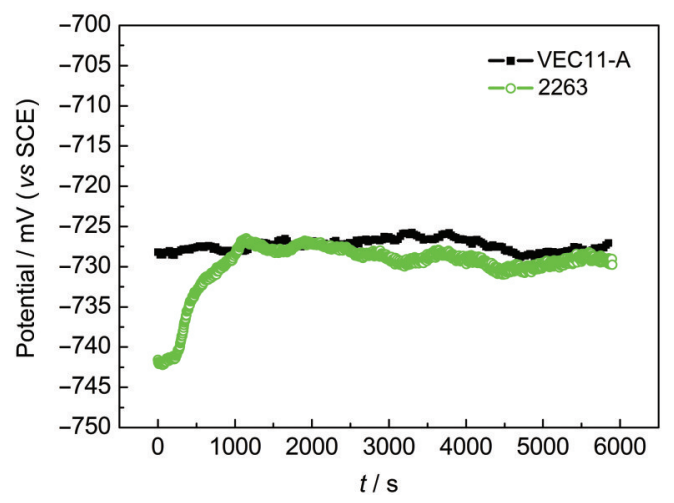

图 $73.5 \%(w) \mathrm{NaCl}$ 溶液中 $\mathrm{Q} 235$ 碳钢自腐蚀测量结果比较

Fig.7 Result comparisons of free-corrosion potential of Q235 carbon steel in 3.5\% (w) $\mathrm{NaCl}$ solution

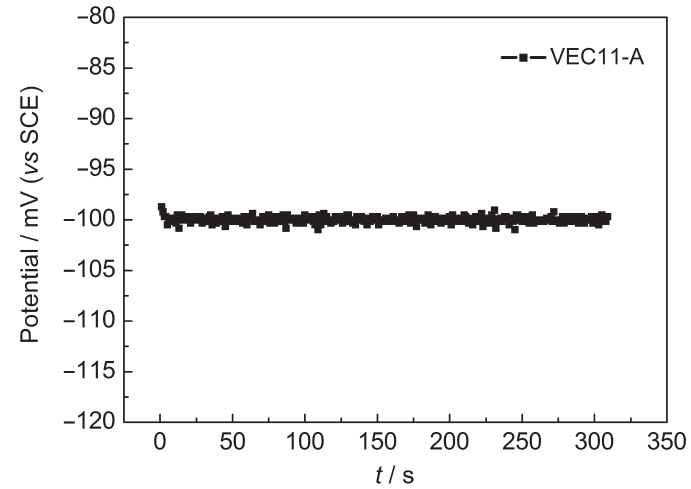

图 8 海水中 $\mathrm{Q} 235$ 碳钢恒电位测量结果比较

Fig.8 Result comparisons of steady potential of Q235 carbon steel in sea water

液、不锈钢电极在 $3.5 \%$ 的 $\mathrm{NaCl}$ 溶液中、不锈钢在缓 蚀剂(3.5\% $\mathrm{HCl}+20 \mathrm{~g} \cdot \mathrm{L}^{-1}$ 六次甲基四胺)中的稳态极 化曲线. 两种仪器的测量结果基本一致, VEC11-A 符合测试要求。

\subsection{4 线性极化曲线测量}

线性极化曲线是腐蚀电化学中常用的线性极 化电阻和腐蚀速率测量方法. 如图 10 所示, 两种仪 器对碳钢 $/ 3.5 \% \mathrm{NaCl}$ 体系的线性极化曲线测量结果 完全一致. 这表明可以使用 VEC11-A 虚拟恒电位仪 测量腐蚀体系的线性极化电阻和腐蚀速率, 这在现 场设备腐蚀速率监测中具有很高的价值.

\subsection{5 动电位扫描测量}

动电位扫描也是腐蚀研究中的常用技术. 图 11 为两种仪器对比测量的碳钢在 $3.5 \% \mathrm{HCl}$ 中的阳极 溶解曲线. 扫描速率为 $0.5 \mathrm{mV} \cdot \mathrm{s}^{-1}$. 由图可以看出, 两种仪器的测量曲线基本重合.

\subsection{6 循环伏安测量}

循环伏安是电化学测量常用技术. 铁氭化钾/亚 铁氰化钾体系具有良好的可逆性, 常用于检测仪器 性能. ${ }^{24}$ 图 12 为两种仪器对比测量的石墨电极在 0.1 $\mathrm{mol} \cdot \mathrm{L}^{-1}$ 铁氰化钾溶液中的循环伏安曲线, 扫描速率 为 $0.5 \mathrm{mV} \cdot \mathrm{s}^{-1}$. 两者的还原峰和氧化峰位置基本相 同, 表明 VEC11-A 虚拟恒电位仪的测量结果是可靠 的.

\subsection{7 钝态极化曲线测量}

金属的针化行为也是腐蚀电化学研究的重要 内容. ${ }^{25}$ 本文采用两种仪器测量了碳钢在 $1 \mathrm{~mol} \cdot \mathrm{L}^{-1}$ 盐酸溶液中的动电位阳极钝化曲线, 扫描速率为 $0.5 \mathrm{mV} \cdot \mathrm{s}^{-1}$. 如图 13 所示, 结果表明, 两条曲线的主 要部分基本一致, 致钝电流和电位完全一致, 钝态 电流和点蚀击破电位等参数基本一致(偏差分别为 

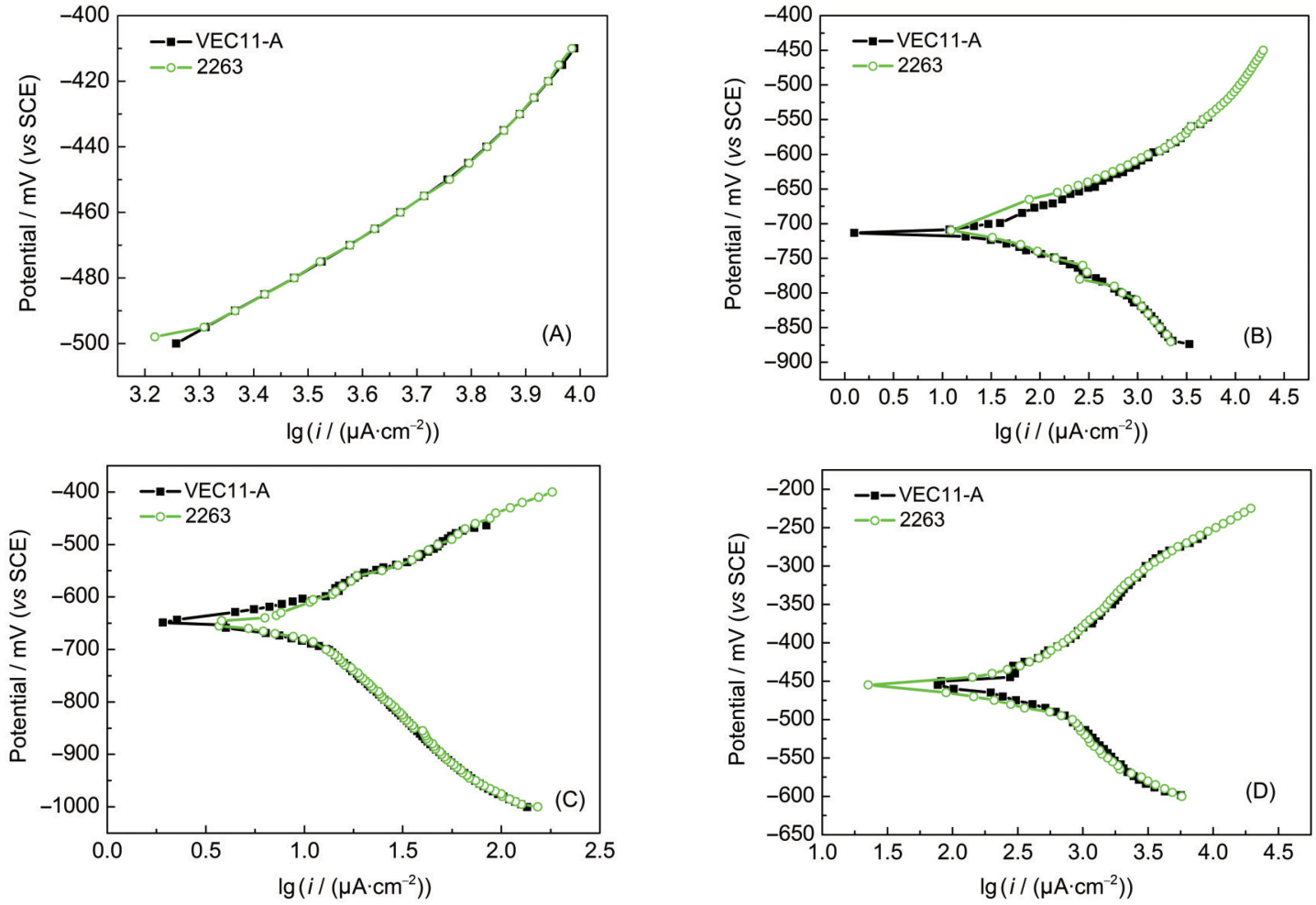

图 9 不同阻抗条件下不同溶液极化曲线结果比较

Fig.9 Result comparisons of polarization curves of different impedances in different solutions scan rate: $0.5 \mathrm{mV} \cdot \mathrm{s}^{-1}$. (A) Q235 carbon steel in $0.5 \mathrm{~mol} \cdot \mathrm{L}^{-1} \mathrm{H}_{2} \mathrm{SO}_{4}$ solution, potential range: $-500--400 \mathrm{mV}$; (B) Q235 carbon steel in $3.5 \%$ $\mathrm{NaCl}$ solution, potential range: $-900--450 \mathrm{mV}$; (C) 304 stainless steel in $3.5 \% \mathrm{NaCl}$ solution, potential range: $-1000--400 \mathrm{mV}$; (D) 304 stainless steel in corrosion inhibitor, potential range: $-1000--200 \mathrm{mV}$

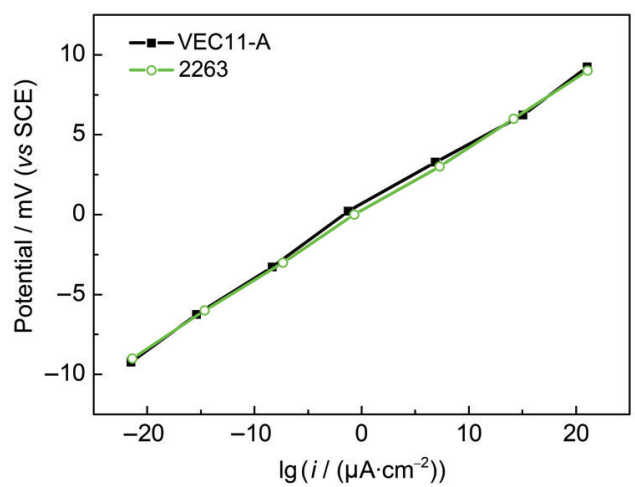

图 $103.5 \% \mathrm{NaCl}$ 溶液中 $\mathrm{Q} 235$ 碳钢线性极化曲线结果比较

Fig.10 Result comparisons of linear polarization curves of Q235 carbon steel in 3.5\% $\mathrm{NaCl}$ solution potential range: $\pm 10 \mathrm{mV}$ relative to open circuit potential; scan rate: $0.5 \mathrm{mV} \cdot \mathrm{s}^{-1}$

$10 \mu \mathrm{A}$ 和 $10 \mathrm{mV}$, 在可接受范围).

\subsection{8 恒电位阶跃响应测量}

恒电位阶跃也是一种常用的电化学测试方法, 本文采用两种仪器测量了 $\mathrm{Q} 235$ 碳钢在 $3.5 \% \mathrm{NaCl}$ 中的恒电位响应曲线, 都在 $300 \mathrm{mV}$ 极化电位下稳 定 $10 \mathrm{~min}$, 然后电位阶跃为 $0 \mathrm{mV}, \mathrm{VEC} 11-\mathrm{A}$ 的数据 采集速率为 $0.2 \mathrm{~s}^{-1}, 2263$ 恒电位仪的数据采集速率

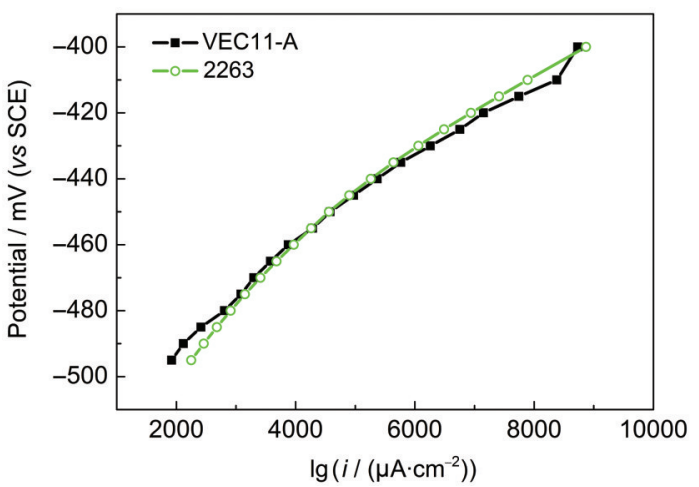

图 $113.5 \% \mathrm{HCl}$ 溶液中 $\mathrm{Q} 235$ 碳钢动电位扫描曲线结果比较

Fig.11 Result comparisons of potentiodynamic polarization curves of Q235 carbon steel in $3.5 \% \mathrm{HCl}$ solution

potential range: $-500--400 \mathrm{mV}$; scan rate: $0.5 \mathrm{mV} \cdot \mathrm{s}^{-1}$

为 $25 \mathrm{~s}^{-1}$. 结果如图 14 所示, 两条曲线的趋势走向基 本一致, 稳定电位基本一致.

\subsection{VEC11-A 虚拟恒电位仪的参数与不足}

VEC11-A 虚拟恒电位仪的基本性能取决于硬 件, 即取决于输入/输出板卡性能. VEC11-A 虚拟恒 电位仪采用的板卡为 NI 公司 M 系列 PCI-6251多功 能采集卡, 是一种中级性能的多功能板卡. 该卡具 


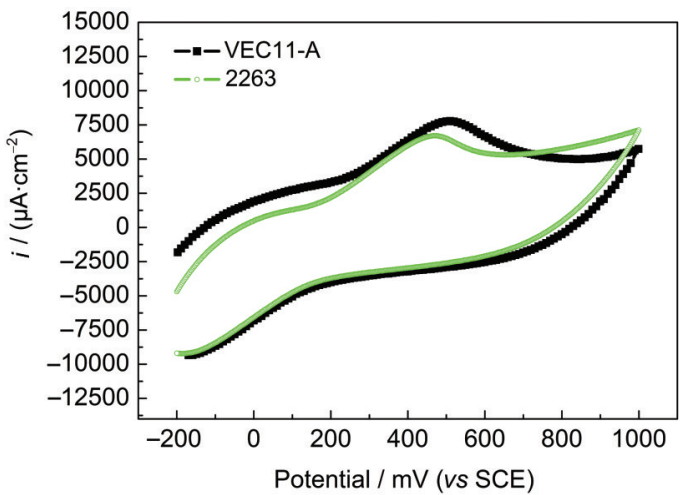

图 $120.1 \mathrm{~mol} \cdot \mathrm{L}^{-1}$ 的 $\mathrm{K}_{3} \mathrm{Fe}(\mathrm{CN})$ 。溶液中石墨电极循环伏安 曲线结果比较

Fig.12 Result comparisons of cyclic voltammetry curves of graphite electrode in $0.1 \mathrm{~mol} \cdot \mathrm{L}^{-1} \mathrm{~K}_{3} \mathrm{Fe}(\mathrm{CN})_{6}$ solution potential range: $-200-1000 \mathrm{mV}$; scan rate: $0.5 \mathrm{mV} \cdot \mathrm{s}^{-1}$

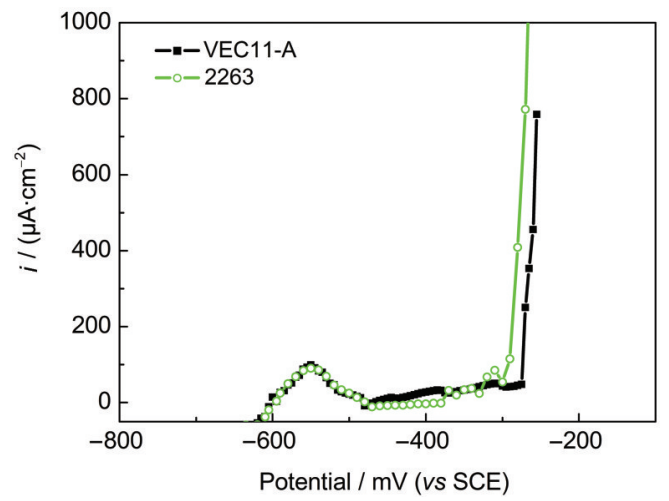

图 $130.1 \mathrm{~mol} \cdot \mathrm{L}^{-1}$ 的 $\mathrm{HCl}$ 溶液中 $\mathrm{Q} 235$ 碳钢动电位阳极 钝化曲线结果比较

Fig.13 Result comparisons of potentiodynamic anode passivation curves of Q235 carbon steel in $0.1 \mathrm{~mol} \cdot \mathrm{L}^{-1}$ HCl solution

potential range: $-650--200 \mathrm{mV}$; scan rate: $0.5 \mathrm{mV} \cdot \mathrm{s}^{-1}$

有 16 bit 分辨率的模拟输入和模拟输出, 最高 1.25 $\mathrm{MS} \cdot \mathrm{s}^{-1}$ 的采样率, 最高模拟输出更新率为 $2.8 \mathrm{MS}$. $\mathrm{s}^{-1}$, 输入阻抗大于 $10^{10} \Omega$. 因此, VEC11-A 虚拟恒电 位仪的主要性能等于和低于 PCI-6251 板卡性能, 其 输入阻抗大于 $10^{10} \Omega$, 最大输入电流约为 $10 \mathrm{~mA}$, 响 应时间约为 $0.5 \mathrm{~s}$. 更换高性能板卡可以显著提高虚 拟恒电位仪性能.

VEC11-A 虚拟恒电位仪的主要特点是分辨率 高, 响应速度适中, 输入阻抗适中. 这一特点在前文 的实际测量中得到了体现, 能够满足常规腐蚀电化 学测量的要求. 其主要不足是输出电流低(小于 10 $\mathrm{mA})$, 不能满足大电流输出的要求. 这一不足可以通 过采用大电流输出的数据采集卡解决, 其升级版 VEP11-B 虚拟恒电位仪正在研制中.

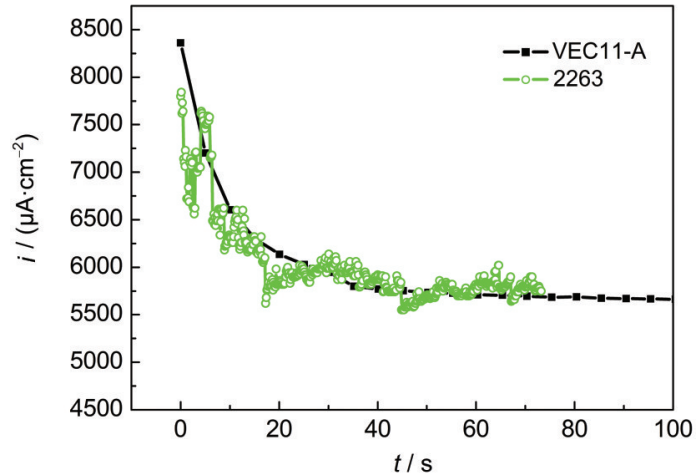

图 $143.5 \% \mathrm{NaCl}$ 溶液中 $\mathrm{Q} 235$ 碳钢恒电位阶跃曲线 结果比较

Fig.14 Result comparisons of constant potential step curves of $\mathrm{Q} 235$ carbon steel in 3.5\% $\mathrm{NaCl}$ solution

\section{4 结 论}

(1) PID 控制能够实现恒电位功能, 基于 PID 控 制的虚拟恒电位仪能够快速稳定控制腐蚀体系中 的工作电极电位达到设定值而不发生自激.

(2) VEC11-A 虚拟恒电位仪的腐蚀电化学测试 系统能够准确可靠地进行腐蚀电位、稳态极化曲 线、线性极化曲线、循环伏安、动电位扫描、恒电位 阶跃等常规腐蚀电化学测量. 测定结果与 2263 商用 恒电位仪一致。

(3) VEC11-A 虚拟恒电位仪腐蚀电化学测试系 统可以借助于采用高性能数据采集卡来满足各级 应用要求.

\section{References}

(1) Yang, L. P.; Li, H. T.; Xiao, K.; Yang, L. An Introduction to the Virtual Instrument Technology; Publishing House of Electronics Industry: Beijing, 2003; pp 2-6. [杨乐平, 李海涛, 肖 凯, 杨 否. 虚拟仪器技术概论. 北京: 电子工业出版社, 2003: 2-6.]

(2) Li, Q. X.; Ren, Y. X. Modern Scientific Instruments 1999, 4 , 10. [李青霞, 任炎晞. 现代科学仪器, 1999, 4, 10.]

(3) Hu, G.; Zhang, S. T. Computers and Applied Chemistry 2006, 23 (5), 465. [胡 舸, 张胜涛. 计算机与应用化学, 2006, 23 (5), 465.]

(4) Pan, G. Y.; Wang, J. Journal of Chinese Society for Corrosion and Protection 2008, 28 (4), 210. [潘国运, 王 佳. 中国腐蚀 与防护学报, 2008, 28 (4), 210.]

(5) Li, H. Q.; Luo, X. B.; Liu, C. X.; Jiang, L. Y.; Cui, D. F.; Cai, X. X. Multi-Channel Electrochemical Detection System Base on LabVIEW. In Information Acquisition, 2004 International Conference on Information Acquisition, Hefei, China, June 21-25, 2004; Yang, Q. D. Ed.; Press of University of Science 
and Technology of China: Hefei, 2005; pp 224-227.

(6) Perusse, P.; Leech, D. Instrumentation Science \& Technology 2000, 28 (1), 59.

(7) Economou, A.; Bolis, S. D.; Efstathoou, C. E.; Volikakis, G. J. Analytica Chimica Acta 2004, 467, 179.

(8) Han, L.; Mai, W.; Wang, S. Y.; Song, S. Z. Corrosion Science and Protection Technology 2004, 16 (1), 50. [韩 否, 买 巍, 王守琰, 宋诗哲. 腐蚀科学与防护技术, 2004, $16(1), 50$.

(9) Han, L.; Song, S. Z. Journal of Chemical Industry and Engineering 2008, 59 (4), 977 . [韩 否, 宋诗哲. 化工学报, 2008, 59 (4), 977.]

(10) Zhong, H. J.; Deng, S. H. Analytical Instrumentation 2009, 2, 1. [钟海军, 邓少华. 分析仪器, 2009, 2, 1.]

(11) Song, S. Z. Corrosion Electrochemical Research Methods; Chemical Industry Press: Beijing, 1988; pp 28-29. [宋诗哲. 腐蚀电化学研究方法. 北京: 化学工业出版社, 1988: 28-29.]

(12) Jin, Q.; Deng, Z. J. Journal of Chongqing Institute of Technology 2008, 22 (5), 91. [金 奇, 邓志杰. 重庆工学院 学报, 2008, $22(5), 91$.]

(13) Jin, Z. Q.; Bao, Q. L. Microcomputer Information 2005, 21 (6), 1. [金志强, 包启亮. 微计算机信息, 2005, $21(6), 1$. ]

(14) Jiang, W.; Yuan, F. Journal of East China Institute of Technology 2004, 12 (4), 395. [江 伟, 袁 芳. 东华理工学 院学报, 2004, 12 (4), 395.]

(15) Wang, W.; Zhang, J. T. Acta Automatica Sinica 2000, 26 (3), 348. [王 伟, 张晶涛. 自动化学报, 2000, 26 (3), 348.]

(16) Ruan, Q. Z. LabVIEW and Me - The Ten-Year-Programming Experience of an Engineer in NI; Beihang University Press: Beijing, 2009; pp 197-246. [阮奇桢. 我和 LabVIEW一个NI工程师的十年编程经验. 北京: 北京航空航天大学
出版社, 2009: 197-246.]

(17) Zhang, K.; Zhou, Z.; Guo, D. LabVIEW Virtual Instrument Engineering Design and Development; National Defence Industry Press: Beijing, 2004; pp 2-6. [张 凯, 周 陬, 郭 栋. LabVIEW 虚拟仪器工程设计与开发. 北京: 国防工业出版社, 2004: 2-6.]

(18) Ye, X. S.; Zhang, G. D.; Gao, B.; Wang, P. Chinese Journal of Scientific Instrument 2005, 26 (10), 1002. [叶学松, 张贵东, 高 波, 王 平. 仪器仪表学报, 2005, 26 (10), 1002.]

(19) Dai, F. P.; Lv, S. Y.; Feng, B. X.; Li, Q. Journal of Lanzhou University 2002, 38 (6), 44. [代富平, 吕淑媛, 冯博学, 李 强. 兰州大学学报, 2002, 38 (6), 44.]

(20) Fan, Z. X.; He, W.; Huo, C. H. Modern Scientific Instruments 2005, (3), 33. [范中晓, 何 为, 霍彩虹. 现代科学仪器, 2005, (3), 33.]

(21) Liu, Y. H. Electrochemical Measurement Technology; Beijing Aviation Institute Press: Beijing, 1987; pp 17-98. [刘永辉. 电化学测试技术. 北京: 北京航空学院出版社, 1987: 17-98.]

(22) Wei, B. M. Metal Corrosion Theory and Application; Chemical Industry Press: Beijing, 1984; pp 170-221. [魏宝明. 金属腐 蚀理论及应用. 北京: 化学工业出版社, 1984: 170-221.]

(23) Zou, Y.; Wang, J. Acta Phys. -Chim. Sin. 2010, 26, 2361. [邹 妍, 王 佳. 物理化学学报, 2010, 26, 2361.]

(24) Fan, X. Z.; Lu, Y. H.; Kong, X. F.; Xu, H. B.; Wang, J. Acta Phys. -Chim. Sin. 2011, 27, 887. [范新庄, 芦永红, 孔祥峰, 徐海波, 王 佳. 物理化学学报, 2011, 27, 887.]

(25) Wang, X. Y.; Wu, Y. X.; Zhang, L.; Yu, Z. Y. Corrosion Science and Protection Technology 2000, 12 (6), 311. [汪轩义, 吴荫 顺, 张 琳, 于正阳. 腐蚀科学与防护技术, 2000, 12 (6), 311.] 